\title{
THE ROLE OF URBAN DESIGN IN ACHIVEING SUSTAINABILITY THROUGH PROMOTION OF SUSTAINABLE BEHAVIORS (CASE STUDY: MELLAT PARK IN TEHRAN, IRAN)
}

\author{
Authors: Maryam Khabazi*, Hooman Foroughmand Araabi**
}

* Science and Research Branch, Islamic Azad University, Tehran, Iran, Email:maryamkhabazi@ymail.com

** Science and Research Branch, Islamic Azad University, Tehran, Iran, Email:hoomanforoughmand@yahoo.com

\begin{abstract}
:
Sustainability is debated in social and economic aspects of urban design but there are few studies on behavioral aspect of it. People with their behavioral patterns have the most significant role in achieving sustainability. The promotion of Behavioral pattern is considered a down-top planning in a country thus any lack in governmental activities enhance the importance of this approach. It means in developing countries where the government does not succeed to achieve sustainability through its top-down planning, considering down-top planning could be efficient. Behavioral patterns shape people consumption of energy and resources and finally determine the consumption rate of city resources. Urban design is one of the branches of knowledge that can obtain the possibility of some circumstances in public spaces of cities for better behaviors so in this article we can suggest some strategies to achieve this target. This article tries to survey how we can achieve patterns of sustainable behavior through some physical changes in urban spaces in the case study of Mellat Park in Tehran, Iran.
\end{abstract}

KEYWORDS: developing countries, urban design, public spaces, physical changes, sustainable behavior, behavioral patterns 


\section{INTRODUCTION}

These days due to all modifications in new world such as industrialization, progress in technology and all achievement of humankind, people think can attain everything they want and consequently they begin to consume resources more and more without considering the limitation of these resources [13]. Little by little, these kinds of behaviors became a part of human characteristic and unsustainable behaviors started to became common. However, after some decades, scientists understood that these resources are restricted and the human must think about ways to reduce their impacts on the earth and its resources. Some of the most significant activities that influence the earth and resources happening in cities because a huge population that live in cities and indeed the most proportion of earth's population live in cities now. So, concentration cities and activities that happen in them are utterly important to abate the negative effects of humankind on the earth.

Urban Design as a field that debates about issues of cities and modify all circumstances of cities through physical changes, can be used as a tool to decrease the adverse impacts of human activities in cities. This has been applied in sustainable urban development approximately during two decades [10], different approaches are available as a result of these efforts, some emphasize on making laws to reduce pollution but some others believe that more democrat approach would be more effective. Nowadays, the role of the public is recognized important in sustainable development in democratic countries but in other countries where democratic organizations are not that much effective their (democratic) principles face problems.

In countries that the government is not concern with the nature and there is no top-down planning to reach sustainable development, the down-top approach would play more important role, on the other hand, while the governmental organizations are not as effective as its equivalents in developed countries, following their principles will not be a good approach, so finding potentials for sustainable development in these societies could be a top priority [12]. Iran for example, has an onerous background for dealing with the lack of energy and water resources, the most beautiful and successful cities in pre-modern Iran were located in this troublesome situation, therefore reaching sustainable development would not be possible without considering cultural potentials. The first step could be reminding this point that it is the human behavior that makes the world polluted so sustainable behaviors studies and sustainable behavior-based design would be very effective in these situations. To make it more vivid we assume that "sustainable behaviors are all kinds of behaviors that reduce the negative effects of behavior on resources, such as better consuming of water and not consuming extravagantly". This article tries to achieve sustainable behavior according to its steps by alteration the built environment and activities.

\section{THE IMPACTS OF PHYSICAL ASSPECTS ON BEHAVIORS}

Behavior is activities that are performed by people, behavioral studies in urban design dates back to (1950's) [3] when psychologists started experiences in real world instead of laboratory the fundamental request for their study was how people perceive and interact with environment and how environment encourages and supports some behaviors, even now these studies are very valuable in Urban Design [3].

In the field of Environmental Psychology, scientists believe that environment could influence or even form the human behaviors but the question that "to some extend the environment could form the behavior" gets different answers [2]. Urban spaces on the other hand are important because these spaces are the places for social activities these places have educational contents especially in socialization for children moreover, these public spaces provide communicative opportunities, So, by urban design we can probably affect human behaviors [1]. In many case-studies, antisocial behaviors are replaced by some convivial activities after alteration in [7]. 
In the past, all endeavors to achieve sustainable behavior relied on increasing education and knowledge of sustainability by assuming that only the knowledge would improve the behaviors. However, although education and increasing knowledge can be effective in creating awareness and in changing attitudes as it will be explained further, numerous studies show that behavior change rarely occurs as a result of simply providing information and when changes do occur they are frequently modest in nature [5].

While debating on resources has been developed in the literature of sustainability and this point that next generations will need these resources has been well developed, emphasis on the next generation behavior seems not be well introduced, promoting sustainable behavior therefore is very essential.

\section{STEPS OF SOCIAL MARKETING IN COMBINATION WITH THE CASE STUDY}

To make the steps clear this article combined the steps with the examples of one case study.

\section{Case study:}

Mellat Park is located in Tehran the capital city of Iran and is one the most important urban parks in Iran. After a fast modernization in Iran during the last century Iranian culture and even identity face a crisis which comes from dichotomy between the modern culture and the Iranian traditional one.

After finding a huge amount of oil in Iran in more than a hundred years ago Iranian economy and way of life change from a nature friendly to a consumer culture, while in pre-modern Iran agriculture were deeply concern with the nature recourses, the oil industry changes the situation for the people and the government, in economy this ended up in oil based economy and in culture it ended up in alienation and following the west culture [11], demographic changes and dramatic increase in city population were results of this change. In this context the government wanted Iranian cities to be like the cities of developed countries. Mellat Park is one of places that had been made for this reason. Mellat park is located near by the Valiasr street which itself is somehow a symbol of modernization process in Iran, the longest street in the middle east with a layer of trees on each side, which was a traditional way of street making in Iran, Mellat park is very well recognized by Terhranian,

On the opposite side of the Mellat Park there are some commercial complexes and houses. While parks first plan respected the topography and natural life, in its small zoo, there is not anything related to sustainability in the park.

Currently, some anti-social behaviors happen around the park but the huge amount of children and youth come to this park that makes a potential for a change [4].

The method for obtaining sustainable behavior in this article is Community-based social marketing, because generally the debate of this article is urban design only the environmental aspects of this method have been used here, in other word the method to improve urban sustainability here is by Community-based social marketing.

Community-based social marketing demonstrates that behavioral changes occur when barriers are removed from an activity and simultaneously activities' benefits are enhanced. This method could be done by its steps, here these steps are introduced by the case study explanations.

Five steps are involved in community-based social marketing [8]:

\subsection{Determining target behaviors}

Like many other methods of planning, target behaviors should be determined. These behaviors are definitely pro-environmental but the target should be achievable and at the same time it is necessary that the level of target's focus remains practical: avoid over-focusing. In the case study, chosen target was that the users of the park do perform sustainable behavior and learn to repeat it in other situations; this means people after attending in the suggested activities become more careful about the nature so the plan strategy is to find ways in which people think the park is for them and they are responsible for it, for this target of course it is vital that the park itself were sustainable, this is the very first step for learning, otherwise all activities would seem 
unreal to the people. Mellat Park is fairly a sustainable place but our critic about it refers to materials, asphalt cement and many unrecyclable materials are in used there and even current constructions use these materials, so replacing them is a case study suggestion.

\subsection{Determining barriers and benefits of these behaviors}

We can divide these barriers into two parts, in order to optimize any action, External and Internal barriers [5].

External barriers are constituted from 2 general factors: 1) Institutional factors which refer to providing a context for action, even if people were keen on doing pro-environmental activities, providing this context is vital. Environment should provide these factors and activities should support it, in the case of Mellat Park, sharing people in activities from watering the trees to collecting rubbish need organizations that should be provided by this plan. 2) Second external barriers are social-cultural factors, people's behavior according to many studies is deeply related to (or as some believe is the result of) the culture, in our case study emphasizing on Iranian nature friendly culture was the application of this level, using old Iranian related word for activities remind many aspects of that culture to the users of space additionally, in educational activities emphasizing on historical and widely recognized stories and using pre-modern physical monuments would both teach the culture and reminds it for the public.

Internal barriers are 1) Motivation, 2) Knowledge, 3) Value, 4) Environmental awareness 5) Emotional involvements. All behaviors occur according to motivations, the question for this part is how to encourage people to do sustainable behavior while some think that knowledge is enough for such situations recent studies show that other aspects are even more important, this part of the method could be very innovational and in the case study using the Fun Theory ${ }^{1}$ was the strategy to encourage people, examples of speaker bins that thank and make some jokes when people throw rubbish there could encourage people to pick up some rubbish for that, the next internal factor is knowledge, despite the fact that experts know the public knowledge is not enough for sustainable behavior, it is important to some extent, it also is important to avoid bombardment people with very professional information. Another factor is values, values strongly forms from childhood, so family is very important for enhancing values. Schools, other groups and even the whole values of society (culture), have their role. The huge number of students and children playing in the Mellat Park make a good potential accordingly. To use this potential making some NGO's could be a good option for the case study. Family activities will make the result even better, another internal factor is environmental awareness, many examples of the natural threats do not involve people, instead of speaking about the ozone layer in the activities, it would be better to speak about species in the park and their illnesses. Trees will get symbolic caretakers who should examine tree's situation these group could take turn to water the tree as well, the knowledge of cooperatives will make them able to recognize other plants illnesses in their city, this will make citizens more careful about the nature as a result, last factor of determining barriers is emotional involvement this makes people more involved with the nature. The case study plan suggests that the empty wall on the south part of the park could be a big canvas for people especially for children to paint there, this would encourage the sense of belonging. Besides, there are bird and animal houses that except their name the atmosphere is like a small zoo, the plans suggest some NGO's for children to bring them closer to animals teach them about their nature and children become able to feed the animals, or even choose names for them and play with them with considering all hygienic aspects, this would involve other sense of children, by this activities children would be less likely to annoy animals in their youth and they would be more care about the nature in their maturity.

\footnotetext{
${ }^{1}$ FUN THEORY is a new way of changing behaviors for the better through some fun activities, with the belief that people inherently enjoy the fun and something as simple as fun is the easiest and most effective way to alter people's behavior for the better. Be for yourself, for the environment, or for something entirely different, the only thing that matters is that it's change for the better. (www.Thefuntheory.com)
} 


\subsection{Developing strategies that reduce the barriers and simultaneously increase the benefits of behaviors}

Environmental solutions in addition to activities would ensure the effectiveness of strategies, using the Fun Theory as it was mentioned, is one of the strategies which encourage people a lot but costs a bit. Giving some activities like watering the flowers will be accepted by people and reduce the cost of management even if this corporations increase costs in short term, in long term by having sustainable behaviors costs would be reduced, so corporation is one the most important strategies in the case study. To let people touch nature the restrictions of going into the grass would be removed, but people should know that they are in charge of it and if they walk on grass too much, they will lose the grass and maybe the park. Of course sort of monitoring is necessary for this strategy but the aim is people themselves monitor and manage the park this would disappear any possible dichotomy between managers and users in the case of pro-environmental activities. Making commitments for people is an objective at this level. The more people think they are responsible for their behavior, the better.

\subsection{Essaying the strategies}

General methods for achieving sustainable behavior need to be examined in a pilot, human behavior is not meticulously predictable. Even before making all places for this case study, some activities could be examined in a small scale. Observing the reaction and behavior of people make a revision possible for the final plan, in our case study due to restricted sources for sampling, only small samples of activity performed and some questionnaires had been completed, the result as it was expected was that people are very keen to attend in these activities but without implication of the plan, all these results are theoretically accepted and real result only obtain from the real plan.

\subsection{Analyzing the strategies while they are implementing}

Further monitoring and observations are significant to improve our knowledge about people's sustainable behavior, what were weaknesses of the plan? How it could be enhanced? What are the results of the plan and which points could be used in other plan? All these questions would get answer if a good system of analyzing and monitoring would be designed.

\section{CONCLUSION: A COHESION PLAN, FINAL PLAN}

A plan for improving sustainable behavior is not complete when it restricts to physical aspects, so for this case study a combination of activities and physical alterations would provide a good environment for target behaviors. Mellat Park, as it is today, has a potential for social activities people gather to each other, this active public place does not have many equivalents in Tehran, this park is a good place for corporative nature friendly activities.

Improving people responsibilities and cares are general goals of this plan, some new buildings for NGO's activities, with recyclable materials, are suggested; using Fun Theory to encourage people to do proenvironmental activities is one of the strategies, and emphasizing on children activities that make them able to become closer to the flora and fauna and getting emotionally involved with the nature by activities are other strategies. Despite the fact that this is very soon to evaluate the result of this plan and this approach, comparing to other activities in public places in Iran, and many other countries, this approach is a good option for many plans. 


\section{REFRENCES}

[1] Altman, Irwin, "The Environment and Social Behavior," Brooks/Cole Publishing Co., 1976.

[2] Betchtel, Robert, and Churchman, Arza, (editors), "Handbook of Environmental Psychology," John Wiley and Sons, 2002.

[3] Cuthbert, Alexander, R., "The Form of Cities, Political Economy and Urban Design.” Oxford, Blackwell, 2006.

[4] Khabazi, Maryam, "The Role of Urban Design in Achieving Sustainability through Promotion of Sustainable Behaviors (Case Study: Mellat Park in Tehran)," Final Thesis on Master of Urban Design, Islamic Azad university of Science and research, Tehran, 2010.

[5] Kollmuss, Anja, Agyeman, Julian, "Mind the Gap: Why do people act environmentally and what are the barriers to pro-environmental behavior?," Routledge Online Publication, 2010.

[6] Lang, Jon, "A Typology of Procedures and Products," Architectural Press, 2005.

[7] Lang, Jon, "Creating Architectural Theory: The Role of the Behavioral Sciences in Environmental Design,” Van Nostrand Reinhold Co., 1987.

[8] McKenzie-Mohr, Doug, Smith's, W., "Fostering Sustainable Behavior: An Introduction to CommunityBased Social Marketing," New Society Publishers, 1999.

[9] Motalebi, Ghasem, "Environmental Psychology,” Fine Arts Magazine, Vol.10, 2001, pp.52-67

[10] Moughtin, Cliff and others, "Urban design Green dimensions," Elsevier, 2003.

[11] Nabavi, Negin, "Intellectual trends in twentieth-century Iran: a critical survey," University Press of Florida, 2002.

[12] Pugh, Cedric, "Sustainable Cities in Developing Countries," Earthscan Publication Ltd, 2000.

[13] Wheeler, S.M., Beatley, T., "The Sustainable Urban Development Reader," Routledge Urban Series, 2008.

[14] www. Thefuntheory.com 